\title{
Projectivity and lifting of Hilbert module maps
}

\author{
by Douglas N. Clark (Athens, Ga.)
}

\begin{abstract}
In a recent paper, Carlson, Foiaş, Williams and the author proved that isometric Hilbert modules are projective in the category of Hilbert modules similar to contractive ones. In this paper, a simple proof, based on a strengthened lifting theorem, is given. The proof also applies to an equivalent theorem of Foiaş and Williams on similarity to a contraction of a certain $2 \times 2$ operator matrix.
\end{abstract}

1. Introduction. One of the obstacles to understanding homological algebra invariants for Hilbert modules is lack of knowledge about projectives in the Hilbert module category (see [3], [1]).

In the work of Douglas, Foiaş and Paulsen, an analogous concept, hypoprojectivity, is defined and it is shown that lifting theorem techniques of operator theory, recast in the Hilbert module setting (see Lemma 0 below), are precisely what is needed to characterize the concept. A result is a proof that the hypoprojective Hilbert modules (over the disk algebra $\mathbb{A}(\mathbb{D})$, for example) are precisely the isometric ones; see [3, Chapter 4]. However, there is no indication that hypoprojectivity is as useful as projectivity in the study of cohomology and other invariants from homological algebra.

In [2], Carlson, Foiaş, Williams and the present author identified the projectives in the category of cramped Hilbert modules over $\mathbb{A}(\mathbb{D})$; the term cramped refers to Hilbert modules similar to contractive ones. In the present note, we obtain one of the main results of [2] (our Theorem 1) in a simpler and more straightforward manner, following the outline of the proof of the characterization of hypoprojectivity in [3, Chapter 4].

Following Douglas and Paulsen [3], we use the term Hilbert module to mean a Hilbert space $H$ together with the action of a function algebra $\mathbb{A}$. If $\mathbb{A}$ is the disk algebra $\mathbb{A}=\mathbb{A}(\mathbb{D})$, properties of the Hilbert module $H$ reflect properties of the operator $T: H \rightarrow H$ defined by $T f=z f$.

1991 Mathematics Subject Classification: Primary 47A20; Secondary 46H25.

Key words and phrases: Hilbert module, lifting theorem, polynomially bounded. 
2. Operator matrices. Theorem 1 below yields a Hilbert-moduletheoretic proof of the following result, known to Foiaş and Williams some years ago, and published in [2].

Let $T_{0}$ and $T_{1}$ be contraction operators on Hilbert spaces $H_{0}$ and $H_{1}, T_{1}$ similar to an isometry. Then the operator

$$
T=\left[\begin{array}{cc}
T_{0} & X \\
0 & T_{1}
\end{array}\right]
$$

is similar to a contraction if and only if there is a bounded operator $L$ : $H_{1} \rightarrow H_{0}$ with $X=L T_{1}-T_{0} L$.

The "if" implication is easy, using the similarity

$$
\left[\begin{array}{cc}
I & -L \\
0 & I
\end{array}\right]\left[\begin{array}{cc}
T_{0} & L T_{1}-T_{0} L \\
0 & T_{1}
\end{array}\right]\left[\begin{array}{cc}
I & L \\
0 & I
\end{array}\right]=\left[\begin{array}{cc}
T_{0} & 0 \\
0 & T_{1}
\end{array}\right] .
$$

For the "only if" part, regard $H_{0}$ and $H_{1}$ as Hilbert modules over $\mathbb{A}(\mathbb{D})$, with multiplication defined by $z f=T_{i} f\left(f \in H_{i}\right)$. Make the direct sum $H_{0} \widehat{\oplus} H_{1}$ into a Hilbert module by defining

$$
z(f, g)=\left(T_{0} f+X g, T_{1} g\right), \quad(f, g) \in H_{0} \widehat{\oplus} H_{1} .
$$

Then $H_{0} \widehat{\oplus} H_{1}$ is cramped if and only if $T$ is similar to a contraction and the exact sequence of Hilbert modules

$$
0 \longrightarrow H_{0} \stackrel{\alpha}{\longrightarrow} H_{0} \widehat{\oplus} H_{1} \stackrel{\beta}{\longrightarrow} H_{1} \longrightarrow 0
$$

$(\alpha f=(f, 0), \beta(f, g)=g)$ splits if and only if there exists $L: H_{1} \rightarrow H_{0}$ such that $f \rightarrow(L f, f)$ is a Hilbert-module map. The latter is equivalent to the relation $X=L T_{1}-T_{0} L$.

But by Theorem 1 of the present note, $H_{1}$, being similar to an isometric module, is projective in the cramped category. It is a matter of elementary homological algebra to show that this implies that (1) splits.

3. Hypoprojectivity. For a Hilbert module $H$ over $\mathbb{A}(\mathbb{D})$, we have automatically

$$
\|p(T) f\|=\|p(z) f\| \leq K\|p\|_{\infty}\|f\|
$$

for $p \in \mathbb{A}(\mathbb{D})$ and $f \in H$; that is, the operator $T$ is polynomially bounded. We shall say that the Hilbert module $H$ has a certain property ( $H$ is contractive, isometric, unitary, etc.) according as the operator $T$ has that property ( $T$ is a contraction, an isometry, a unitary operator, etc.). By the same convention, a Hilbert module $K$ is a (minimal, isometric) dilation of $H$ if $K \supseteq H$, as Hilbert spaces, and the operator $T^{\prime}$ of multiplication by $z$ on $K$ is a (minimal, isometric) dilation of the corresponding operator $T$ on $H$. In this case, the orthogonal projection $q: K \rightarrow H$ is a Hilbert-module map (i.e. $q$ carries the action of $\mathbb{A}(\mathbb{D})$ on $K$ into the action of $\mathbb{A}(\mathbb{D})$ on $H)$. The term isometric 
dilation Hilbert module is a compromise on the term Shilov dominant of [3], where more general function algebras are considered.

In the terminology just adopted, the lifting theorem of Sz.-Nagy and Foiaş may be stated as follows.

Lemma 0. Let $H_{0}$ and $H_{1}$ be contractive Hilbert modules, with $K_{0}$ and $K_{1}$ their minimal isometric dilation modules, and let $\Phi: H_{1} \rightarrow H_{0}$ be a Hilbert-module map. Then there is a Hilbert-module map $\widetilde{\Phi}: K_{1} \rightarrow K_{0}$ with $\|\widetilde{\Phi}\|=\|\Phi\|$ and such that the diagram

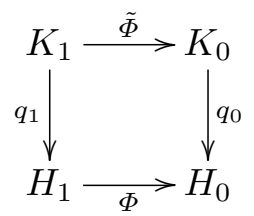

commutes, where $q_{0}$ and $q_{1}$ are the orthogonal projections.

See Douglas and Paulsen [3, Theorem 4.12]; we have, in this version, avoided the use of their term Shilov resolution.

The following theorem of Douglas and Foiaş can be stated as "Shilov modules are hypoprojective", and its proof can be based upon Lemma 0 (see [3, Theorems 4.11 and 4.16]).

TheORem 0. Suppose $\mathcal{P}, H_{0}$ and $H_{1}$ are Hilbert modules over $\mathbb{A}(\mathbb{D})$ with $\mathcal{P}$ isometric and $H_{i}$ contractive, $i=0,1$. Suppose $\varphi: H_{1} \rightarrow H_{0}$ and $\Phi:$ $\mathcal{P} \rightarrow H_{0}$ are Hilbert-module maps with $\varphi$ partially isometric and surjective. Then there exists a Hilbert-module map $\tilde{\Phi}: \mathcal{P} \rightarrow H_{1}$ with $\|\widetilde{\Phi}\|=\|\Phi\|$ and making

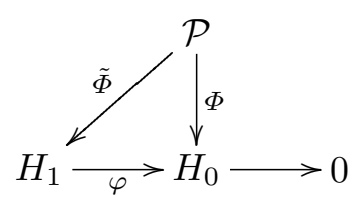

commute.

The principal device in the proof of Theorem 0, besides Lemma 0, is Lemma 1 below, in the case where $\varphi$ is a partial isometry; the two diagrams are put together as in the proof of Lemma 2 below.

4. Projectivity. Our first lemma is a modest generalization of the main step in the proof of Theorem 4.16 of Douglas and Paulsen [3].

LEMMA 1. Suppose $H_{0}$ and $H_{1}$ are contractive Hilbert modules over $\mathbb{A}(\mathbb{D})$ and $\varphi$ is a Hilbert-module map of $H_{1}$ onto $H_{0}$. Then there exists a Hilbertmodule map $\eta: K_{0} \rightarrow H_{1}$, where $K_{0}$ is the minimal isometric dilation 
module of $H_{0}$, such that

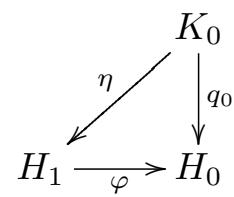

commutes and $\|\eta\| \leq\left\|\left[\left.\varphi\right|_{(\operatorname{ker} \varphi)^{\perp}}\right]^{-1}\right\|$.

Proof. Write $H_{1}=\operatorname{ker} \varphi \oplus H_{1}^{\prime}$ and let $\psi: H_{0} \rightarrow H_{1}^{\prime}$ be the inverse of the restriction of $\varphi$ to $H_{1}^{\prime}$.

Give $H_{1}^{\prime}$ the compressed Hilbert module structure inherited from $H_{1}$. That is, let $\mathbb{A}(\mathbb{D})$ act on $H_{1}^{\prime}$ by

$$
p\left(z^{\prime}\right) f=P p(z) f
$$

for $p \in \mathbb{A}(\mathbb{D})$ and $f \in H_{1}^{\prime}$, where $P$ is the projection of $H_{1}$ and $H_{1}^{\prime}$. Then $\psi$ is a Hilbert-module map because, for $g \in H_{0}$,

$$
\psi z g=\psi z \varphi \psi g=\psi \varphi z \psi g=\psi \varphi P z \psi g=z^{\prime} \psi g .
$$

Now let $K_{1}^{\prime}$ denote the minimal isometric dilation module of $H_{1}^{\prime}$. We can arrange to have

$$
K_{1}^{\prime} \subset K_{1}
$$

where $K_{1}$ is the minimal isometric dilation module of $H_{1}$; indeed, $K_{1}^{\prime}$ is isomorphic to $\mathbb{A}(\mathbb{D}) \cdot H_{1}^{\prime}$ (in the multiplication of $K_{1}$ ).

Now the lifting theorem (Lemma 0) implies the existence of a Hilbertmodule map $\Psi: K_{0} \rightarrow K_{1}^{\prime}$ with $\|\Psi\|=\|\psi\|$ and with

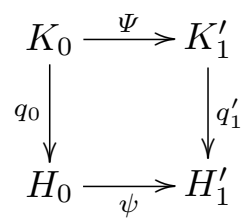

commuting.

For $f \in K_{0}$, we therefore have $q_{1}^{\prime} \Psi f=\psi q_{0} f$, or $\varphi q_{1}^{\prime} \Psi f=q_{0} f$. If we set $\eta=q_{1}^{\prime} \Psi$ and replace $H_{1}^{\prime}$ by $H_{1}=H_{1}^{\prime} \oplus \operatorname{ker} \varphi$, the lemma follows.

The following lemma shows how to put the diagrams in Lemmas 0 and 1 together. The result contains Theorem 0 .

LEMMA 2. If $\mathcal{P}$ is an isometric Hilbert module, $H_{0}$ and $H_{1}$ are contractive Hilbert modules, $\varphi: H_{1} \rightarrow H_{0}$ is a surjective Hilbert-module map and $\Phi: \mathcal{P} \rightarrow H_{0}$ is a Hilbert-module map, then there exists a Hilbert-module 
$\operatorname{map} \widetilde{\Phi}: \mathcal{P} \rightarrow H_{1}$ with

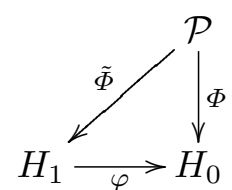

commuting and $\|\widetilde{\Phi}\| \leq\|\Phi\| \cdot\left\|\left[\left.\varphi\right|_{(\operatorname{ker} \varphi)^{\perp}}\right]^{-1}\right\|$.

Proof. Let $K_{0}$ be the minimal isometric dilation module of $H_{0}$. By the lifting theorem (Lemma 0), there exists $\Phi^{\prime}: \mathcal{P} \rightarrow K_{0}$ making

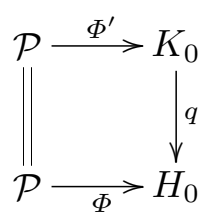

commute and with $\left\|\Phi^{\prime}\right\|=\|\Phi\|$. By Lemma 1, we see that

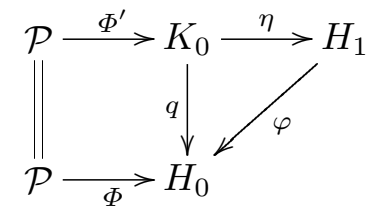

commutes and $\|\eta\| \leq\left\|\left[\left.\varphi\right|_{(\operatorname{ker} \varphi)^{\perp}}\right]^{-1}\right\|$. Setting $\widetilde{\Phi}=\eta \Phi^{\prime}$ completes the proof.

The following theorem is equivalent to Corollary 3.3 of [2]: Hilbert modules similar to isometric Hilbert modules are projective in the category of cramped Hilbert modules. But since Theorem 1 comes from the lifting theorem, we can also give a bound on the operator constructed.

If $L$ is a bounded, invertible operator on Hilbert space, we denote by $k(L)$ the positive constant

$$
k(L)=\|L\| \cdot\left\|L^{-1}\right\| .
$$

TheOREm 1. Let $\mathcal{P}, H_{0}$, and $H_{1}$ be Hilbert modules with $\mathcal{P}$ similar to an isometric Hilbert module and $H_{0}$ and $H_{1}$ cramped. That is, let

$$
T=L S L^{-1} \quad \text { and } \quad T_{i}=L_{i} S_{i} L_{i}^{-1}, \quad i=0,1,
$$

where $T$ is multiplication by $z$ on $\mathcal{P}, T_{i}$ is multiplication by $z$ on $H_{i}, i=0,1$, $S$ is an isometry and $S_{i}$ is a contraction, $i=0,1$. Suppose further that $\varphi: H_{1} \rightarrow H_{0}$ and $\Phi: \mathcal{P} \rightarrow H_{0}$ are Hilbert-module maps with $\varphi$ surjective. Then there exists a Hilbert-module map $\widetilde{\Phi}: \mathcal{P} \rightarrow H_{1}$ with

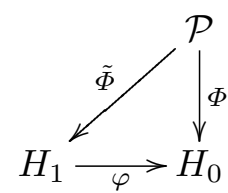


commuting and with

$$
\|\widetilde{\Phi}\| \leq k(L) k\left(L_{0}\right) k\left(L_{1}\right)\left\|\left[\left.\varphi\right|_{(\operatorname{ker} \varphi)^{\perp}}\right]^{-1}\right\| \cdot\|\Phi\| .
$$

Proof. Let $\mathcal{P}^{\prime}$ denote the Hilbert space $\mathcal{P}$ with the $\mathbb{A}(\mathbb{D})$-action

$$
p\left(z^{\prime}\right) \cdot f=p(S) f, \quad p \in \mathbb{A}(\mathbb{D}) .
$$

Similarly, for $i=0,1$, let $H_{i}^{\prime}$ denote $H_{i}$ with

$$
p\left(z^{\prime}\right) \cdot f=p\left(S_{i}\right) f, \quad p \in \mathbb{A}(\mathbb{D}) .
$$

Then we have the diagram

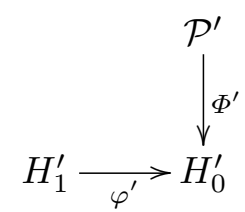

where $\varphi^{\prime}=L_{0}^{-1} \varphi L_{1}$ and $\Phi^{\prime}=L_{0}^{-1} \Phi L$. By Lemma 2, we can lift $\varphi^{\prime}$ in the above diagram to $\widetilde{\Phi}^{\prime}: \mathcal{P}^{\prime} \rightarrow H_{1}^{\prime}$. Then, observing that $L: \mathcal{P}^{\prime} \rightarrow \mathcal{P}$, $L_{0}: H_{0}^{\prime} \rightarrow H_{0}$ and $L_{1}: H_{1}^{\prime} \rightarrow H_{1}$ are Hilbert-module maps, and checking norms, the theorem follows.

The existence of isometric dilations for contractive Hilbert modules over $\mathbb{A}(\mathbb{D})$ provides projective resolutions in the category of cramped Hilbert modules. For details of their use, as well as connections between Theorem 1 and the famous problem of the existence of noncramped Hilbert modules, one may consult [2].

\section{References}

[1] J. F. Carlson and D. N. Clark, Cohomology and extensions of Hilbert modules, J. Funct. Anal. 128 (1995), 278-306.

[2] J. F. Carlson, D. N. Clark, C. Foias and J. P. Williams, Projective Hilbert $\mathbb{A}(\mathbb{D})$-modules, New York J. Math. 1 (1994), 26-38.

[3] R. G. Douglas and V. I. Paulsen, Hilbert Modules over Function Algebras, Longman Scientific and Technical, New York, 1989.

Department of Mathematics

University of Georgia

Athens, Georgia 30602

U.S.A.

E-mail: dnc@alpha.math.uga.edu 